\title{
Signed zero forcing number and controllability for a networks system with a directed hypercube
}

\author{
Gufang Mou ${ }^{1}$, and Qiuyan Zhang ${ }^{1,2, *}$ \\ ${ }^{1}$ College of Applied Mathematics, Chengdu University of Information Technology, China \\ ${ }^{2}$ School of Mathematical Sciences, University of Electronic Science and Technology of China
}

\begin{abstract}
The controllability for complex network system is to find the minimum number of leaders for the network system to achieve effective control of the global networks. In this paper, the problem of controllability of the directed network for a family of matrices carrying the structure under directed hypercube is considered. The relationship between the minimum number of leaders for the directed network system and the number of the signed zero forcing set is established. The minimum number of leaders of the directed networks system under a directed hypercube is obtained by computing the zero forcing number of a signed graph.
\end{abstract}

Keywords: Directed networks system, Signed zero forcing, Signed graph, Minimum number of leaders.

\section{Introduction}

In the real information age, many dynamical systems exist in the form of complex network. To ensure the normal operation of these systems, it is necessary to effectively control the whole system. The controllability for complex network system was studied by using a graph topology, such as [1-6], the necessary or sufficient conditions for controllability were shown. With the help of a network system graph topology: the vertices in a graph represent the node, and the edges represent the connection relationship between the nodes. Using the maximum matching algorithm of bipartite graph, unmatched nodes are used as driving nodes to get the minimum set of leaders [10]. However, the maximum matching set of the network is not unique, and the resulting minimum driving node set is not unique. In [7], controllability analysis was carried out by the notion of zero forcing sets for digraph. In 2011, L. Hogben et al. studied the controllability for complex network system by using the minimum rank theory of graph. A lower bound of communication complexity is determined by using the minimum rank of symbol pattern. The zero forcing set of undirected graph or digraph is applied to the analogy possibility between development networks, linear control system theory, Lie algebra theory of quantum system.

* Corresponding author: zqy1607@,cuit.edu.cn 
In this paper, the problem of controllability of the directed network for a family of matrices carrying the structure under directed hypercube is considered. The relationship between the minimum number of leaders for the directed network system and the number of the signed zero forcing set is established, the minimum number of leaders of the directed networks system under a directed hypercube is obtained by computing the zero forcing number of a sign graph.

\section{Definitions}

Firstly, we will introduce the definitions of matrices and graphs.

The graph of $\mathrm{A}$ is a digraph, denoted by $\Gamma$ with vertices $\{1,2, \ldots, n\}$ and $\operatorname{arcs}\{(i, j)$ : $\left.a_{i j} \neq 0\right\}$. The order of $\Gamma$, denoted by $|\Gamma|$, is the number of vertices of $\Gamma$. A matrix having entries in $\{+,-, 0\}$ is called A sign pattern matrix. For a given simple directed graph $G$, the vertex set of $G$ is a nonempty set and is denoted by $V(G)$. The arc set of $G$, denoted by $E(G)$, is a subset of $V \times V$, and $(i, i) \notin E$ for all $i \in V(G)$. The cardinality of a given set $V$ is denoted by $|V|$. Also we use $|G|$ to denote in short the cardinality of $V(G)$.

A signed digraph is a digraph $\Gamma=(V, E$, sign $)$, which consists of a digraph $(V, E)$ together with a sign function sign $: E \rightarrow\{1,-1\}$, denoted $\operatorname{sign}(e), e \in E$, that is, the edges (arcs) of $\Gamma$ have been signed positive or negative by sign.

Definition 2.1. In a digraph $\Gamma$, if the directed edge is $i \rightarrow j$ or $i \leftarrow j$, then the sign of edge $(i, j)$ is "+" or "-". If there is no edge between $i$ and $j$, then it is marked 0 . For an $n \times n$ sign pattern $P$, the graph of $P$ is a signed digraph $\Gamma$.

Definition 2.2 [7]. If the form is

$$
\dot{x}=X x+U u,
$$

then it is called the controllability analysis of linear input/state systems, where $x \in R^{n}$ is the state and $u \in R^{m}$ is the input with the distinguishing feature that the matrix $X$ is associated with a given graph $G$ and the matrix $U$ encodes the vertices (often called leaders) through which external inputs are applied.

Definition 2.3 [7]. For a given graph $G$, the minimum number of leaders rendering all systems (1) is controllable denoted by $l_{\min }(G)$, that is,

$$
l_{\min }(G)=\min \left\{\left|V_{L}\right|:\left(G ; V_{L}\right) \text { is controllable, } V_{L} \subseteq V(G)\right\} .
$$

\section{Main results}

In [7], the relationship between the minimum number of leaders for the directed network system and the number of the signed zero forcing set is established. In this section, we will change a digraph for a directed network for a family of matrices carrying the structure to a signed digraph, and show the signed zero forcing set for a signed digraph. A one-to-one correspondence between the minimum number of leaders for the directed network system and the number of the signed zero forcing set is established.

Zero forcing is played on the vertices in a graph, whose vertices are coloured black or white. The coloring change rule is to change the color of a white vertex w to black; in this case we say $u$ forces $v$. Then a color-change rule is applied until no more changes are possible. This rule is different in a simple undirected graph or a directed graph or a signed digraph. We will introduce two the zero forcing rules.

Rule 1 [8] Consider a graph $G$ and colour each of its vertices black or white.

If a vertex $u$ is white and a vertex $v$ is black, $u$ is a neighbour of $v$, and $u$ is the only white neighbour of $v$, then change the colour of $u$ to black and continue the iterative procedure and all the vertices of $G$ are blackened. 
The set $S$ is called a zero forcing set if this procedure, starting from a graph where only the vertices in $S$ are black, leads to a graph where all vertices are black.

Example 3.1. A zero forcing process is shown in Figure 1, 2, 3. Consider that the vertices 1,2 of graph in Figure 1 are initially coloured black. It is clear that $1 \rightarrow 4$ in Figure 1 by applying rule 1 . Consequently, $2 \rightarrow 3$ in Figure 2 . Thus $\{1,2\}$ is a zero forcing set.

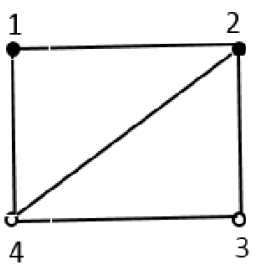

Fig. 1. Zero forcing process

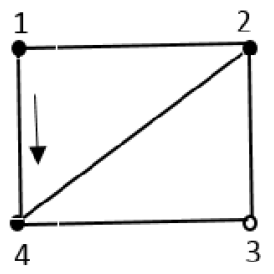

Fig. 2. Zero forcing process

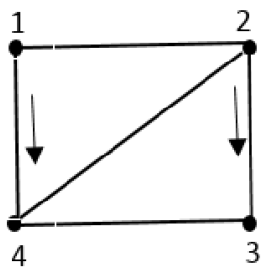

Fig. 3. Zero forcing process

Rule 2 [9] Let $u$ be a vertex of $P$ such that either $u$ is black or $u$ is white and $P_{u u} \neq$ ? Let

Define

$$
W=\{w \mid w \text { is white } \wedge w \rightarrow u\} \cup\{u\}
$$

and

$$
W_{+}=\left\{w \in W \mid m(w)=P_{u w}\right\}, W_{-}=\left\{w \in W \mid m(w) \neq P_{u w}\right\}
$$

$$
W_{*}=\{w \in W \mid m(w)=*\}
$$

where $\mathrm{m}(\mathrm{w})$ is denoted the sign + or - of a marked vertex; otherwise we will write $\mathrm{m}(\mathrm{w})=$ *.

(a) If $W=\{w\}$, color $w$ black.

(b) If either $W_{+}=W$ or $W_{-}=W$, color all vertices in $W$ black.

(c) If $W \neq \varnothing, W_{l(s)}=\emptyset$, and $W_{*}=\{w\}$, mark $w$ with $P_{u w} \cdot l(s)$, where the sign function $l$ is defined by $\imath(+)=-(l(-)=+)$ and $s$ is used as indices taking values in $\{+,-\}$.

(d) If no white vertices are marked and $u$ is white, then mark $u$ with + .

The signed zero forcing number of $P, Z_{ \pm}(P)$ is the size of the minimum forcing set in signed zero forcing rule.

Lemma 3.2 [7]. For a given graph $G$, then the minimum number of leaders rendering all systems of controllability $l_{\min }(G)=\mathrm{Z}(G)$.

Example 3.3. Let $\Gamma$ be a digraph for a simple directed network system (see Figure 4). First, we change $\Gamma$ to a signed graph. $\Gamma$ is corresponded to a sign pattern $P=\left[\begin{array}{cccc}? & + & + & + \\ - & ? & + & - \\ - & - & ? & 0 \\ - & + & 0 & ?\end{array}\right]$.

Firstly, we colour the vertices 1,2 black $(S=\{1,2\})$ and mark vertex 3 with + . Next let $u=1$, we obtain that $W_{+}=\{2,3\}$ and $W_{*}=\{4\}$ by applying Rule $2(c)$. Therefore the vertex 4 can be marked with $P_{34} \cdot l(+)=-$. We also apply Rule $2(c)$ to vertex $2, W_{+}=\{3,4\}$. Finally, the vertices 3, 4 are blackened. So we can obtain $\mathrm{Z}_{ \pm}(G)=2$. Thus, the minimum number of leaders rendering for a simple directed network system under $\Gamma$ is two by Lemma 2.5 . 


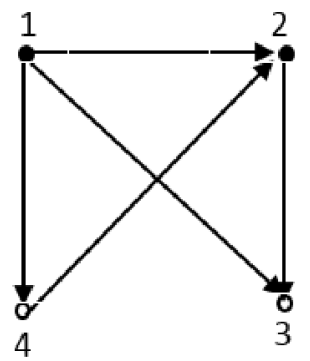

Fig. 4. Simple directed network system.

Definition 3.4 [8]. Let $G$ and $H$ be two graphs. If the graph with vertex set $V(G) \times V(\mathrm{H})$ such that $(u, v)$ is adjacent to $\left(u^{\prime}, v^{\prime}\right)$ if and only if (1) $u=u^{\prime}$ and $v v^{\prime} \in E(H)$ or (2) $v=v^{\prime}$ and $u u^{\prime} \in E(G)$ is called the Cartesian product of $G$ and $H$, denoted by $G \square H$.

Definition 3.5 [8]. A complete graph is a graph $K_{n}=\left(\left\{v_{1}, v_{2}, \cdots, v_{n}\right\}, E\right)$ such that $K_{n}=\left\{\left\{v_{i}, v_{j}\right\}, 1 \leq i, j \leq n\right\}$.

If $Q_{1}=K_{2}, \quad Q_{2}=K_{2} \square K_{2}\left(Q_{2}=Q_{1} \square K_{2}\right), Q_{3}=Q_{2} \square K_{2}, \cdots, Q_{n+1}=Q_{n} \square K_{2}$, then $Q_{n}$ is defined $n$th hypercube. Clearly $\left|Q_{n}\right|=2^{n}$.

Definition 3.6. If all the edges of $Q_{n}$ is directed, then $Q_{n}$ is called directed hypercube.

Lemma 3.7 [8]. For any graphs $G, H, Z(G \square H) \leq \min \{\mathrm{Z}(G)|H|, \mathrm{Z}(H)|G|\}$.

Theorem 3.8. Let $Q_{n}$ be directed hypercube, then $\mathrm{Z}_{ \pm}\left(Q_{n}\right) \leq 3 \times 2^{n-3}$, for all $n \geq 3$.

Proof. The proof is by induction on $n$.

Let $n=3, Q_{3}$ be the directed hypercube of dimension 3 (see Figure 5).

is a sign pattern whose digraph is $Q_{3}$.

$$
P=\left[\begin{array}{cccccccc}
? & + & 0 & + & 0 & 0 & 0 & + \\
- & ? & + & 0 & 0 & 0 & + & 0 \\
0 & - & ? & + & 0 & + & 0 & 0 \\
- & 0 & - & ? & - & 0 & 0 & 0 \\
0 & 0 & 0 & + & ? & + & 0 & - \\
0 & 0 & - & 0 & - & ? & + & 0 \\
0 & - & 0 & 0 & 0 & - & ? & - \\
- & 0 & 0 & 0 & + & 0 & + & ?
\end{array}\right]
$$

We will show that $Z_{ \pm}\left(Q_{3}\right)=3$ in according to rule 2 . Firstly, we colour the vertices 1,2 , 8 black $(S=\{1,2,8\})$ and seed vertex 7 with + . Next let $u=8$, we can obtain $W_{-}=\{7\}$ and $W_{*}=\{5\}$ by applying Rule $2(c)$. Therefore the vertex 5 can be marked with $P_{85} \cdot l(-)=+$, that is, $\mathrm{m}(5)=-$. We also use Rule $2(c)$ to vertex $2, W_{+}=\{5\}, W_{*}=\{3\}$, and the vertex 3 can be with $P_{23} \cdot l(+)=-$, that is, $\mathrm{m}(3)=-$. Because the vertex 4 is only white vertex, it can be blackened by applying Rule 1 . Finally, let $u=4, p_{43}=p_{45}=-, W_{+}=\{3,5\}$, then the vertices 3 , 5 are blackened.

The vertices 6, 7 are white and can be blacken by applying Rule 1. So we can obtain $\mathrm{Z}_{ \pm}\left(Q_{3}\right)=3$. 
Let $n=4, Q_{4}=Q_{3} \square K_{2}$, then $\mathrm{Z}_{ \pm}\left(Q_{4}\right) \leq 3 \times 2=3 \times 2^{4-3}$ in according to Lemma 2.10 .

Suppose $n=k, \mathrm{Z}_{ \pm}\left(Q_{k}\right) \leq 3 \times 2^{k-3}$ is true.

When $n=k+1, Q_{k+1}=Q_{k} \square K_{2}$. We can obtain $\mathrm{Z}_{ \pm}\left(Q_{k+1}\right) \leq \mathrm{Z}_{ \pm}\left(Q_{k+1}\right) \times\left|K_{2}\right| \leq 3 \times 2^{(k+1)-3}$ using the induction hypothesis.

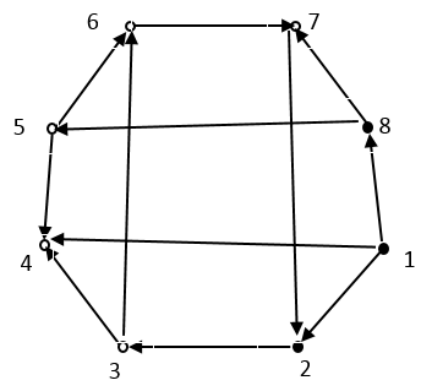

Fig. 5. Proof

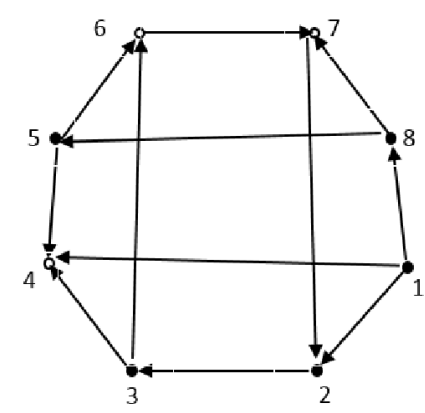

Fig. 6. Proof.

Theorem 3.9. For a directed networks system under a directed hypercube $Q_{n}$, the upper bound of $l_{\min }\left(Q_{n}\right)$ is $3 \times 2^{n-3}$.

The work was supported by National Natural Science Foundation of China (Grant No. 12101090).

\section{References}

1. M. K. Camlibel, S. Zhang, and M. Cao. Comments on 'Controllability analysis of multi-agent systems using relaxed equitable partitions'. International Journal of Systems, Control and Communications, 4(1/2):72-75, 2012.

2. M. Egerstedt, S. Martini, M. Cao, M. K. Camlibel, and A. Bicchi. Interacting with networks: How does structure relate to controllability in single-leader, consensus network? Control Systems Magazine, 32(4): 66-73, 2012.

3. S.Martini, M. Egerstedt, and A. Bicchi. Controllability analysis of multiagent systems using relaxed equitable partitions. International Journal of Systems, Control and Communications, 2(1/2/3): 100-121, 2010.

4. A. Rahmani, M. Ji, M. Mesbahi, and M. Egerstedt. Controllability of multi-agent systems from a graph theoretic perspective. SIAM Journal on Control and Optimization, 48(1): 162-186, 2009.

5. A. Y. Yazicioglu, W. Abbas, and M. Egerstedt. A tight lower bound on the controllability of networks with multiple leaders. In Proc. of the 51st IEEE conference on Decision and Control, pages 1978-1983, 2012.

6. S. Zhang, M. K. Camlibel, and M. Cao. Controllability of diffusively coupled multiagent systems with general and distance regular topologies. In Proc. of the 50th IEEE conference on Decision and Control and 2011 European Control Conference, pages 759-764, 2011.

7. Monshizadeh N, Zhang S, Camlibel M K. Zero Forcing Sets and Controllability of Dynamical Systems Defined on Graphs. IEEE Transactions on Automatic Control, 2014, 59(9):2562-2567. 
8. AIM Minimum Rank-Special Graphs Work Group. Zero forcing sets and the minimum rank of graphs. Linear Algebra and its Applications, 2008, 428(7): 1628-1648.

9. Goldberg F, Berman A. Zero forcing for sign patterns. Linear Algebra and its Applications, 2014, 447: 56-67.

10. Trefois M, Delvenne J C. Zero forcing number, constrained matchings and strong structural controllability. Linear Algebra and its Applications, 2015, 484: 199-218. 